\title{
Possible Narrow Dips in the High-Energy Proton-Proton Scattering at Large $|t|$.
}

T. SAWADA

Department of Physics and Astrophysies, University of Colorado - Boulder, Colo.

(Nuovo Cimento, $55 \mathrm{~A}, 342(1968)$ )

There is an error in the plotting of the data of Fig. $1 a$ ). The point at $\sqrt{--t}=3.20 \mathrm{GeV} / \mathrm{c}$ and $R=0.86$ should be changed to a point at $\sqrt{-t}=3.20 \mathrm{GeV} / \mathrm{c}$ and $R=1.01$.

Double-Pion Photoproduction and Isobaric Model Below $1 \mathrm{GeV}$.

I. Giannini and A. Santroni

Istituto Nazionale di Fisica Nucleare - Sezione di Genova

Istituto di Fisica dell'Università - Genova

(Nuovo Cimento, 55 A, 359 (1968))

At the end of the caption of Fig. 5 of this paper please read:

a) $\varphi^{*}=0^{\circ}$ and $180^{\circ}$, b) $\varphi^{*}=233^{\circ}$. 